05

\title{
Влияние структурных факторов на прочность и электропроводность объемных наноструктурных медных сплавов
}

\author{
() Р.Г. Чембарисова, И.В. Александров, А.М. Ямилева
}

Уфимский государственный авиационный технический университет, 450008 Уфра, Россия

e-mail: chroza@yandex.ru

(Поступило в Редакцию 30 января 2018 г.)

\begin{abstract}
Представлены результаты анализа структурных параметров, обеспечивающих повышение прочности и понижение электросопротивления сплавов системы $\mathrm{Cu}-\mathrm{Cr}$, подвергнутых интенсивной пластической деформации (ИПД) и старению. Модельные расчеты удельного сопротивления и прочностных характеристик выполнены на примере сплава $\mathrm{Cu}-0.5 \mathrm{wt} . \% \mathrm{Cr}$, в котором в результате ИПД сформировано наноструктурное состояние. Сопоставление с экспериментальными значениями продемонстрировало адекватность выбранного подхода для описания исследованных состояний. Показано, что формирование наноструктурных состояний с высокой прочностью и низким электросопротивлением реализуется благодаря одновременному влиянию нескольких факторов, вклад каждого из которых может варьироваться в зависимости от параметров обработки материала.
\end{abstract}

DOI: $10.21883 / J T F .2019 .02 .47069 .46-18$

\section{Введение}

Чистые металлы и сплавы широко используются в качестве проводников электрического тока. Однако от металлических материалов, как правило, требуется целый ряд параметров с определенными значениями. Например, актуальным является достижение высокой прочности материала в сочетании с его высокой пластичностью. С точки зрения практического применения востребованными являются проводники, обладающие высокой прочностью и высокой электропроводностью. Поэтому актуальной остается задача сочетания высоких прочностных свойств с функциональными физическими свойствами.

Интенсивная пластическая деформация (ИПД) [1] позволяет вводить в структуру образца различные наноструктурные элементы, такие как дислокации, располагающиеся преимущественно в границах зерен (ГЗ), частицы вторичной фазы [2], дефекты упаковки [3], сегрегации примесных атомов вдоль ГЗ [2], нанодвойники [4], которые приводят к повышению прочности. В то же время продемонстрирована возможность одновременного повышения как прочностных, так и пластических свойств [4,5], а также целенаправленного управления физическими свойствами наноматериалов, в частности, их электропроводностью $[5,6]$.

Увеличение прочности металлических материалов за счет введения в структуру различных препятствий движению дислокаций [2,4] сопровождается снижением их электропроводности [5-7]. Возрастание удельного сопротивления металлов и сплавов, подвергнутых ИПД, обусловлено рассеянием электронов на различных дефектах кристаллической решетки, введенных в процессе деформирования материала, примесных атомах и тепловых колебаниях решетки. Чем выше концентрация структурных дефектов и температура металлических материалов, тем выше их удельное электрическое сопротивление $\rho[7,8]$.

В качестве проводников тока широко используется медь [9]. Поэтому актуальной задачей является не только повышение ее прочности, но и сохранение высокой электропроводности. Большое влияние на физикомеханические свойства меди оказывают атомы легирующих химических элементов [6]. В этой связи особый интерес представляют исследования, выполненные на сплавах одной системы с различным содержанием компонентов, а значит, и с различной энергией дефекта упаковки [3].

Увеличение содержания легирующего элемента приводит к формированию в результате ИПД меньшего размера зерна и повышению прочностных свойств материала [3]. При этом в работах [6,10-12] показано, что увеличение электропроводности может быть достигнуто в результате очищения матрицы от растворенных атомов и модификации частиц вторичной фазы в процессе старения [6]. Авторами работы [6] было показано, что дополнительное старение при $450^{\circ} \mathrm{C}$ в течение $0.5 \mathrm{~h}$ образцов сплава $\mathrm{Cu}-0.5 \mathrm{Cr}-0.12 \mathrm{Ag}$, подвергнутых интенсивной пластической деформации кручением (ИПДК), привело к увеличению прочности на растяжение от $480 \mathrm{MPa} \mathrm{после}$ ИПДК до $840 \mathrm{MPa} \mathrm{в} \mathrm{результате} \mathrm{последующего} \mathrm{старения.}$ В то же время электропроводность увеличилась от $30 \%$ IACS (International Annealed Copper Standard) до 85\% IACS в результате выделения наночастиц Cr из раствора. Высокая прочность без значительного понижения электропроводности была достигнута в результате уменьшения размера зерна и упрочнения сплава дисперсными частицами.

Авторы работы [5] подвергли сплав $\mathrm{Cu}-0.5$ wt.\% Cr холодной прокатке после четырех проходов равноканаль- 
ного углового прессования (РКУП) и последующему старению в течение $1 \mathrm{~h}$ при $450^{\circ} \mathrm{C}$, что привело к увеличению пластичности до $22 \%$. Прочность при растяжении составила $554 \mathrm{MPa}$. Электропроводность достигла значения 84\% IACS. В то же время после четырех проходов РКУП пластичность была равна $\approx 13 \%$, прочность $460 \mathrm{MPa}$, электропроводность составила $\approx 35 \%$ IACS. Возросшую прочность образцов сплава в данном случае авторы объяснили измельчением зерна и выделением частиц вторичной фазы, препятствующих скольжению дислокаций. Оказалось, что электропроводность не зависит от числа проходов. В результате был сделан вывод о том, что внесенные в процессе РКУП дислокации практически не влияют на электропроводность сплава в отличие от растворенных в матрице атомов.

Имеется большое количество экспериментальных работ, например, [5,6,10-12], в которых продемонстрирована возможность получения методами ИПД наноструктурных материалов, в которых высокая прочность сочетается с высокой электропроводностью. В то же время актуальной остается задача получения количественных оценок влияния различных наноструктурных элементов на электропроводность металлических материалов на основе соответствующих моделей рассеяния электронов внесенными в процессе ИПД дефектами кристаллической структуры. Целью настоящей работы является анализ на основе аналитических моделей влияния элементов микроструктуры, таких как растворенные в матрице атомы примеси, дислокации, частицы вторичной фазы на прочность и электропроводность сплавов системы $\mathrm{Cu}-\mathrm{Cr}$, в том числе подвергнутых ИПД и последующей термообработке.

\section{1. Методика моделирования}

\section{1. Механическая прочность металлических материалов}

Механическая прочность сплавов системы $\mathrm{Cu}-\mathrm{Cr}$ pacсчитывалась с учетом вклада сопротивления со стороны кристаллической решетки, дислокационного упрочнения, упрочнения растворенными в матрице примесными атомами и частицами вторичных фаз.

\subsection{1. Напряжение Пайерлса-Набарро}

Согласно модели Пайерлса [13], энергия активации скольжения дислокации на единицу ее длины из одного положения в другое идентичное положение, минуя положение с максимальной энергией, равна

$$
\Delta W \approx\left(G b^{2} / 2 \pi K\right) \exp (-2 \pi y / d)
$$

где $y$ - расстояние, на котором сдвиг вдоль плоскости скольжения, возникающий для аккомодации дислокации, не превышает половины своего максимального значения, равного $\pm b / 2, K=1$ для винтовой дислокации и $K=(1-v)$ - для краевой дислокации, $d-$ расстояние между атомами в направлении скольжения, которое принято равным значению вектора Бюргерса матрицы $b, v-$ коэффициент Пуассона, $G$ - модуль сдвига матрицы. Тогда сила, действующая на единицу длины дислокации $F_{L}$, будет иметь вид

$$
F_{L}=\tau_{P} b \approx(G b / K) \exp (-2 \pi y / d),
$$

где $\tau_{P}$ - напряжение сдвига, $y=x / K, x-$ расстояние между соседними плоскостями, в которых происходит скольжение. Тогда минимальное напряжение $\sigma_{P}$ (напряжение Пайерлса-Набарро), необходимое для движения дислокаций в потенциальном поле бездефектной кристаллической решетки, будет описываться выражением

$$
\sigma_{P} \approx(M G / K) \exp (-2 \pi x / K d),
$$

где $M$ - фактор Тейлора. Выражение (1) является приближенным и может дать лишь порядок величины $\sigma_{P}$ для простых решеток.

\subsection{2. Дислокационное упрочнение}

Дислокации, лежащие в плоскостях, пересекаемых движущимися дислокациями и упругие взаимодействия дислокаций с дислокациями, лежащими в плоскостях, параллельных плоскостям скольжения, приводят к появлению сопротивления их движению, которое можно оценить по формуле [14]

$$
\sigma_{d i s l}=M \alpha G b \sqrt{\rho_{t o t}},
$$

где $\alpha-$ коэффициент, который может изменяться в интервале от 0.089 до 0.5 [15], $\rho_{t o t}$ - скалярная плотность дислокаций.

\subsection{3. Твердорастворное упрочнение металлических материалов}

Твердорастворное упрочнение обусловлено взаимодействием дислокаций с примесными атомами [16]. При этом основной вклад в прочностные характеристики вносят трение при движении дислокаций и закрепление дислокаций примесными атомами. Появление трения при движении дислокаций связано с активностью двух механизмов. Первый обусловлен несоответствием размеров атомов примеси и матрицы, характеризуемым параметром $\varepsilon_{b}$ :

$$
\varepsilon_{b}=\frac{1}{b} \frac{d b}{d c},
$$

где $\varepsilon_{b}-$ скорость расширения решетки при изменении концентрации, являющаяся мерой несоответствия атомных размеров основного и легирующего элементов, $c$ атомная концентрация растворенных атомов. Второй несоответствием модулей упругости атомов и матрицы, характеризуемый параметром $\varepsilon_{G}$,

$$
\varepsilon_{G}=\frac{1}{G} \frac{d G}{d c}
$$


Согласно данным, представленным в работе [17], считалось, что твердорастворное упрочнение $\sigma_{c}$ в медных сплавах определяется сопротивлением движению винтовых дислокаций. Возможно, это связано с тем, что микроскопическая текучесть обусловлена значительным ростом подвижных дислокаций. Поскольку винтовые дислокации способны размножаться при поперечном скольжении, то им принадлежит в этом значительная роль. Напряжение течения в условиях взаимодействия с винтовой дислокацией в медных сплавах описывается выражением

$$
\sigma_{c} \approx \frac{M G \varepsilon_{s}^{3 / 2} c^{n}}{760}
$$

где $\varepsilon_{s}=\varepsilon_{G}^{\prime}+3 \varepsilon_{b}, \varepsilon_{G}^{\prime}=\varepsilon_{G}\left(1+\left|\varepsilon_{G}\right| / 2\right)$. В случае растворения элементов, вызывающих плавное упрочнение, приращение прочности пропорционально их концентрации в степени $n=1$ или $n=1 / 2$. Пропорциональность твердорастворного упрочнения величине $c^{1 / 2}$ встречается в большей части экспериментальных данных, однако такая зависимость не является общей закономерностью [17]. Практически зависимость упрочнения от концентрации не отличается от линейной [18]. В то же время пропорциональность $\varepsilon_{s}^{3 / 2}$ хорошо согласуется с экспериментальными результатами [17].

\subsection{4. Упрочнение частицами вторичной фазы}

Упрочнение материала возможно когерентными и некогерентными частицами вторичной фазы.

Наличие в матрице частиц когерентной фазы, кристаллическая решетка которой плавно переходит в кристаллическую решетку матрицы, приводит к появлению в матрице упругих искажений. При этом упрочнение оценивают в соответствии с подходом, предложенным Моттом и Набарро [19], который учитывает возможность формирования дислокаций несоответствия и поверхностей раздела между частицей и матрицей, появляющихся при перерезании когерентных частиц движущимися дислокациями [20]. Небольшие когерентные частицы могут перерезаться дислокациями. Максимальный радиус частиц, которые перерезаются дислокациями, равен $r_{\max }=\alpha b / 2 \xi$, где $\xi-$ функция, характеризующая несоответствие между параметрами решеток матрицы и выделения [15]. Упрочнение когерентными частицами вторичной фазы можно оценить по формуле $[21,22]$ (рис. 1)

$$
\sigma_{s e c}=M \chi \xi^{3 / 2} G\left(\frac{R f_{s e c}}{\alpha b}\right)^{1 / 2},
$$

где $R$ - радиус частиц, $f_{s e c}-$ объемная доля частиц вторичной фазы, $\chi-$ коэффициент, который может иметь значения из интервала 2-3. Обычно принимают значение $\chi=2.6[15,22]$.

С увеличением размера частиц требуются значительно большие напряжения для их перерезания. Когда частицы становятся большими и расстояния между ними увеличиваются, становится возможным явление

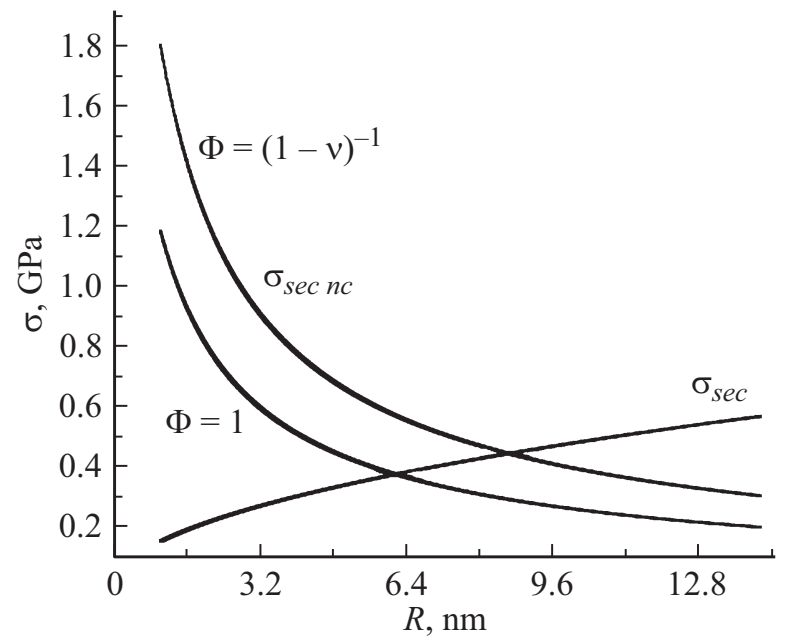

Рис. 1. Упрочнение когерентными частицами $\sigma_{s e c}$ и некогерентными частицами $\sigma_{\text {sec nc }}$ в зависимости от радиуса частиц вторичной фазы $R$ при $f_{\text {sec }}=0.0057, Q=0.81$.

огибания частиц дислокациями. Большие некогерентные частицы - частицы, обособленные от матрицы межфазной границей раздела и отдаленные на значительные расстояния одна от другой, как правило, обходятся дислокациями с образованием петель. Упрочнение материала некогерентными частицами может быть описано в рамках модели, предложенной Е. Орованом, которая учитывает огибание частиц движущимися дислокациями [23]:

$$
\sigma_{\text {sec } n c}=Q M \frac{G b}{21 \pi(|L-D|)} \Phi \ln \left(\left|\frac{L-D}{4 b}\right|\right),
$$

$D=2 R$ - средний размер частиц, $\Phi=1$ в случае винтовой дислокации, $\Phi=(1-v)^{-1}-$ для краевой дислокации, $L-$ расстояние между частицами, $Q-$ параметр, учитывающий неравномерность распределения частиц в матрице, равный 0.81-0.85 [24]. С увеличением размера частиц напряжение, требуемое для обхода частиц дислокациями, уменьшается (рис. 1). Расстояние между частицами можно оценить по формуле $L=R \cdot \sqrt[3]{4 \pi / 3 f_{s e c}}$.

\section{2. Электропроводимость металлических материалов}

Удельное сопротивление металлических материалов зависит от наличия дефектов кристаллической решетки, на которых происходит рассеяние электронов. Электроны могут рассеиваться и на колебаниях атомов в узлах кристаллической решетки, амплитуда которых и соответственно сечение рассеяния электронов зависят от температуры. С понижением температуры вклад в удельное сопротивление тепловых колебаний атомов решетки стремится к нулю. Существенными оказываются лишь рассеяния электронов на дефектах кристаллического строения материала, не зависящие от температуры. 


\subsection{1. Электропроводность при высоких и промежуточных температурах}

Удельная проводимость $\sigma$ металлов чувствительна к температуре [8]. Для определения зависимости удельного сопротивления $\rho_{T}$ от температуры $T$, значительно превышающей температуру Дебая $\Theta$, можно применить классическое уравнение [25], в котором вместо средней скорости теплового движения электронов использована скорость Ферми $u_{F}$ :

$$
\sigma_{T}(T \gg \Theta)=\frac{1}{\rho_{T}}=\frac{n e^{2}}{m_{e} u_{F} n_{a} s_{T}} .
$$

В качестве сечения рассеяния $s_{T}$ электронов, образующих газ фермионов, принято сечение рассеяния на тепловых колебаниях кристаллической решетки, которое в приближении Эйнштейна прямо пропорционально температуре $T$ металлического материала

$$
s_{T}=\frac{2 \pi T \hbar^{2}}{m_{a} k_{B} T_{E}^{2}} .
$$

Здесь $a-$ концентрация (объемная) атомов или ионов кристаллической решетки матрицы, $m_{e}-$ масса электрона, $n$ - концентрация электронов, $e-$ величина заряда электрона, $m_{a}$ - масса атома или иона матрицы, $\hbar$ - постоянная Планка, $k_{B}$ - постоянная Больцмана, $T_{E}$ - температура Эйнштейна. Для $\mathrm{Cu} T_{E}=200 \mathrm{~K}[26]$.

В промежуточной области температур удельное сопротивление $\rho_{T}$ пропорционально $T^{5}$, благодаря вкладу электрон-фононного взаимодействия. Такая температурная зависимость соответствует закону БлохаГрюнайзена $[8,27,28]$

$$
\sigma_{T}=\frac{1}{\rho_{T}}=\sigma_{T}(T \gg \Theta) \cdot\left(\frac{\hbar \omega_{D}}{k T}\right)^{4},
$$

где $\omega_{D}$ - частота Дебая, которая может быть определена из условия $\hbar \omega_{D}=k_{B} \Theta$. Температура Дебая $\Theta$ для крупнокристаллической $\mathrm{Cu}$, согласно таблицам, приведенным в сборнике [29], равна 310, 335, 315-330, 315 K. Установлено, что в наноструктурных состояниях металлических материалов она понижается [1]. Примем значение $\Theta=315 \mathrm{~K}$. Тогда при температуре $T=300 \mathrm{~K}$ с учетом того, что в $\mathrm{Cu}$ концентрация электронов примерно равна концентрации атомов: $n \approx n_{a}$, удельная проводимость, согласно формуле (5), оказалась равной $\sigma_{T} \approx 6.04 \cdot 10^{7} 1 / \Omega \cdot \mathrm{m}$. Удельное сопротивление соответственно $-\rho_{T \mathrm{Cu}} \approx 1.7 \cdot 10^{-8} \Omega \cdot \mathrm{m}$.

Удельное сопротивление чистой электротехнической $\mathrm{Cu}$ при комнатной температуре, согласно экспериментальным данным, равно $\rho_{T \mathrm{Cu}} \approx(1.724-1.8) \cdot 10^{-8} \Omega \cdot \mathrm{m} \quad$ [9]. Теоретическая формула (5) позволяет получать результаты, сопоставимые с экспериментальными значениями.

\subsection{2. Электропроводность металлических материалов при низких температурах}

При сверхнизких температурах вклад теплового движения атомов кристаллической решетки в удельное сопротивление металла стремится к нулю с приближением температуры к абсолютному нулю. В результате удельное сопротивление определяется главным образом рассеянием электронов дефектами кристаллической решетки. Упругие процессы рассеяния испытывают лишь электроны, которые в импульсном пространстве располагаются вблизи поверхности Ферми. Для электронов, находящихся в глубине сферы Ферми, процессы рассеяния исключаются в силу принципа запрета Паули. Длина волны де Бройля будет одной и той же при всех температурах $T$. Соответственно сечение рассеяния электронов на структурных дефектах не будет зависеть от температуры. В результате температурная зависимость удельного сопротивления при низких температурах будет стремиться к постоянному значению.

Электрическое сопротивление реальных металлов определяется рядом одновременно действующих процессов рассеяния электронов на различных дефектах кристаллической решетки. Поэтому полагая, что они независимы, удельное электрическое сопротивление материала определяют в виде суммы их вкладов, согласно правилу Маттисена [30-32]:

$$
\rho=\frac{m_{e} u_{F}}{n_{a} e^{2}} \sum_{i} C_{V i} S_{i} .
$$

Здесь $C_{V i}$ - концентрация (объемная) дефекта $i, S_{i}$ сечение рассеяния электрона на дефекте. Проанализируем вклад различных дефектов кристаллической решетки в электропроводность металлических материалов на примере сплавов системы $\mathrm{Cu}-\mathrm{Cr}$.

\subsubsection{1. Вклад твердого раствора}

Введение примесного атома в кристаллическую решетку приводит к изменению её потенциала, что можно представить как появление кулоновского поля точечного заряда, имеющего порядок элементарного заряда $q$. Рассеяние на растворенном атоме происходит в результате взаимодействия свободного электрона с этим потенциалом $[8,33]$. Большое количество свободных электронов в металле приводит к экранированию измененного потенциала в результате их перераспределения. Зависимость от расстояния $r$ потенциала $\varphi$ точечного заряда $q$, окруженного электронным газом Ферми, можно найти в результате решения уравнения Пуассона для потенциала

$$
\Delta \varphi=\frac{q}{\varepsilon_{0}}\left(n(\mathbf{r})-n_{0}\right),
$$

где $n_{0}-$ равновесная плотность электронов, $n(\mathbf{r})-$ локальная плотность электронов, $\varepsilon_{0}$ - электрическая постоянная, $\varepsilon_{0} \approx 8.85 \cdot 10^{-12} \mathrm{~F} \cdot \mathrm{m}^{-1}$. В рамках приближения Томаса-Ферми [34], согласно которому энергия 


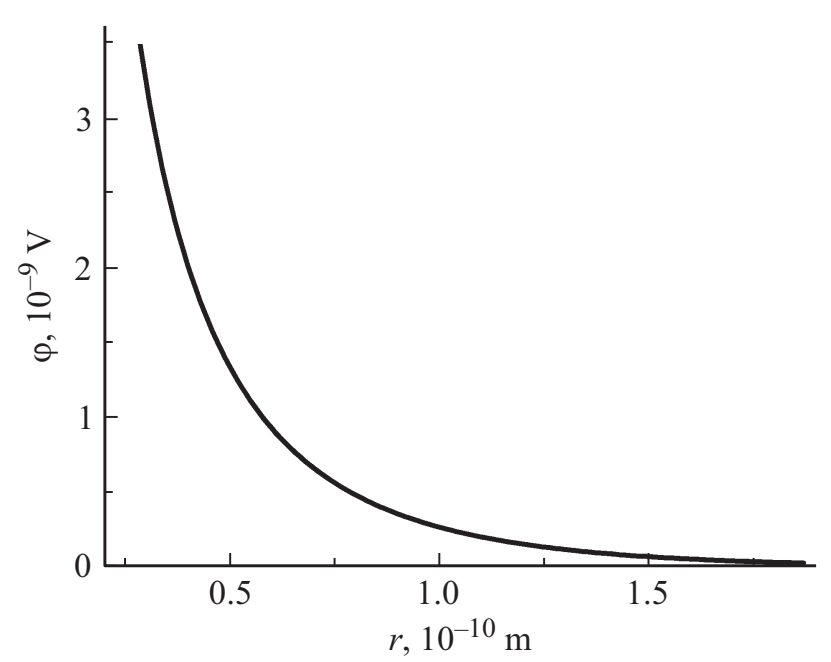

Рис. 2. Зависимость потенциала $\varphi$ от расстояния $r$ от точечного заряда.

Ферми определяется плотностью электронов, на большом расстоянии от примесного атома его заряд полностью экранирован, и химический потенциал в состоянии равновесия будет равен

$$
\begin{aligned}
\mu=E_{F}-q \varphi(\mathbf{r}) & =-\frac{\hbar^{2}}{2 m}\left(3 \pi^{2} n\right)^{2 / 3}-q \varphi(\mathbf{r}) \\
& =\frac{\hbar^{2}}{2 m}\left(3 \pi^{2} n_{0}\right)^{2 / 3}
\end{aligned}
$$

Из последнего равенства следует, что для ферми-газа справедливо выражение

$$
\begin{aligned}
q \varphi(\mathbf{r}) & =E_{F}(n(\mathbf{r}))-E_{F}(n(\mathbf{r}))=\frac{d E_{F}}{d n}\left(n(\mathbf{r})-n_{0}(\mathbf{r})\right) \\
& =\frac{2}{3} \frac{E_{F}}{n_{0}}\left(n(\mathbf{r})-n_{0}(\mathbf{r})\right)
\end{aligned}
$$

С учетом соотношения (8) уравнение Пуассона (7) может быть представлено в виде

$$
\Delta \varphi=\frac{q^{2}}{\varphi_{0}} \frac{3 n_{0}}{2 E_{F}} \varphi=\delta^{2} \varphi, \quad \delta^{2}=\frac{q^{2}}{\varphi_{0}} \frac{3 n_{0}}{2 E_{F}} .
$$

Решением уравнения (9) является потенциал, быстро убывающий с расстоянием (рис. 2):

$$
\varphi=q \frac{e^{-\delta r}}{r} .
$$

Таким образом, кулоновский потенциал точечного заряда экранируется на расстояниях $r>1 / \delta$, где $1 / \delta \approx 0.554 \cdot 10^{-10} \mathrm{~m}$ (рис. 2). В качестве длины экранирования было принято значение $r=3 / \delta$.

Термообработка, которой подвергаются деформированные образцы сплава $\mathrm{Cu}-\mathrm{Cr}$, приводит к распаду твердого раствора и выделению дисперсно-упрочняющих частиц вторичной фазы, что является причиной убыли концентрации растворенных в матрице атомов.
Предполагая, что частицы вторичной фазы имеют сферическую форму радиуса $R$, число атомов в одной частице можно определить из формулы

$$
N_{\mathrm{Cr}}=\frac{4 \pi \rho_{\text {dens } \mathrm{Cr}} R^{3}}{3 \mu_{\mathrm{Cr}} m_{0}},
$$

где $m_{0}$ - атомная единица массы. Число частиц в образце равно

$$
N_{s e c}=\frac{3 V_{s} f_{s e c}}{4 \pi R^{3}} .
$$

$V_{s}$ - объем образца. Число атомов во всех частицах вторичной фазы соответственно определится следующим образом:

$$
N_{\mathrm{Cr} s e c}=N_{\mathrm{Cr}} N_{\text {sec }}=\frac{V_{s} \rho_{\text {dens } \mathrm{Cr}} f_{\text {sec }}}{\mu_{\mathrm{Cr}} m_{0}}
$$

Исходная объемная концентрация растворенных атомов равна

$$
C_{V \mathrm{Cr}}=\frac{W \rho_{\text {dens } \mathrm{Cr}}}{\mu_{\mathrm{Cr}} m_{0}},
$$

где $\mu_{\mathrm{Cr}}, \mu_{\mathrm{Cu}}, \rho_{\text {dens } \mathrm{Cr}}, \rho_{\text {dens } \mathrm{Cu}}$ - относительные атомные массы и плотности $\mathrm{Cr}$ и $\mathrm{Cu}$ соответственно, $W$ - концентрация атомов $\mathrm{Cr}$ по весу. В то же время объемная концентрация атомов $\mathrm{Cr}$, выделившихся из раствора в частицы вторичной фазы, равна

$$
C_{V \mathrm{Cr} s e c}=\frac{\rho_{\text {dens } \mathrm{Cr}} f_{s e c}}{\mu_{\mathrm{Cr}} m_{0}} .
$$

Тогда в зависимости от объемной доли частиц вторичной фазы $f_{s e c}$ атомная концентрация $\mathrm{Cr}$ в растворе с учетом равенств (11) и (12) может быть представлена в виде

$$
\begin{aligned}
\Delta C_{\mathrm{Cr}} & =\frac{\Delta C_{V \mathrm{Cr}}}{n_{a}}=\frac{C_{V \mathrm{Cr}}-C_{V \mathrm{Cr} s e c}}{n_{a}} \\
& =\frac{\mu_{\mathrm{Cu}}}{\mu_{\mathrm{Cr}}}\left(W-\frac{\rho_{\text {dens } \mathrm{Cr}}}{\rho_{\text {dens } \mathrm{Cu}}} f_{\text {sec }}\right)
\end{aligned}
$$

где $n_{a}=N_{\mathrm{Cu}} / V_{s}=\rho_{\text {dens } \mathrm{Cu}} / \mu_{\mathrm{Cu}} m_{0}-$ объемная концентрация атомов $\mathrm{Cu}$. Соответственно удельное сопротивление, обусловленное рассеянием электронов на растворенных атомах $\mathrm{Cr}$, изменяется по закону

$$
\rho_{\mathrm{Cr}}=\frac{m_{e} u_{F}}{e^{2}} \Delta C_{\mathrm{Cr}} S_{\mathrm{Cr}}
$$

В данном случае сечение рассеяния принято равным $S_{\mathrm{Cr}}=\pi r^{2}$, где $r-$ радиус экранирования кулоновского поля примесного атома электронами. Графики зависимости концентрации $\Delta C_{\mathrm{Cr}}$ атомов $\mathrm{Cr}$ в матрице от объемной доли $f_{\text {sec }}$ частиц вторичной фазы (13), а также вклада $\rho_{\mathrm{Cr}}(14)$, растворенных в матрице атомов $\mathrm{Cr}$ в удельное сопротивление сплава $\mathrm{Cu}-\mathrm{Cr}$ в зависимости от их атомной концентрации $\Delta C_{\mathrm{Cr}}$ представлены на рис. 3. 

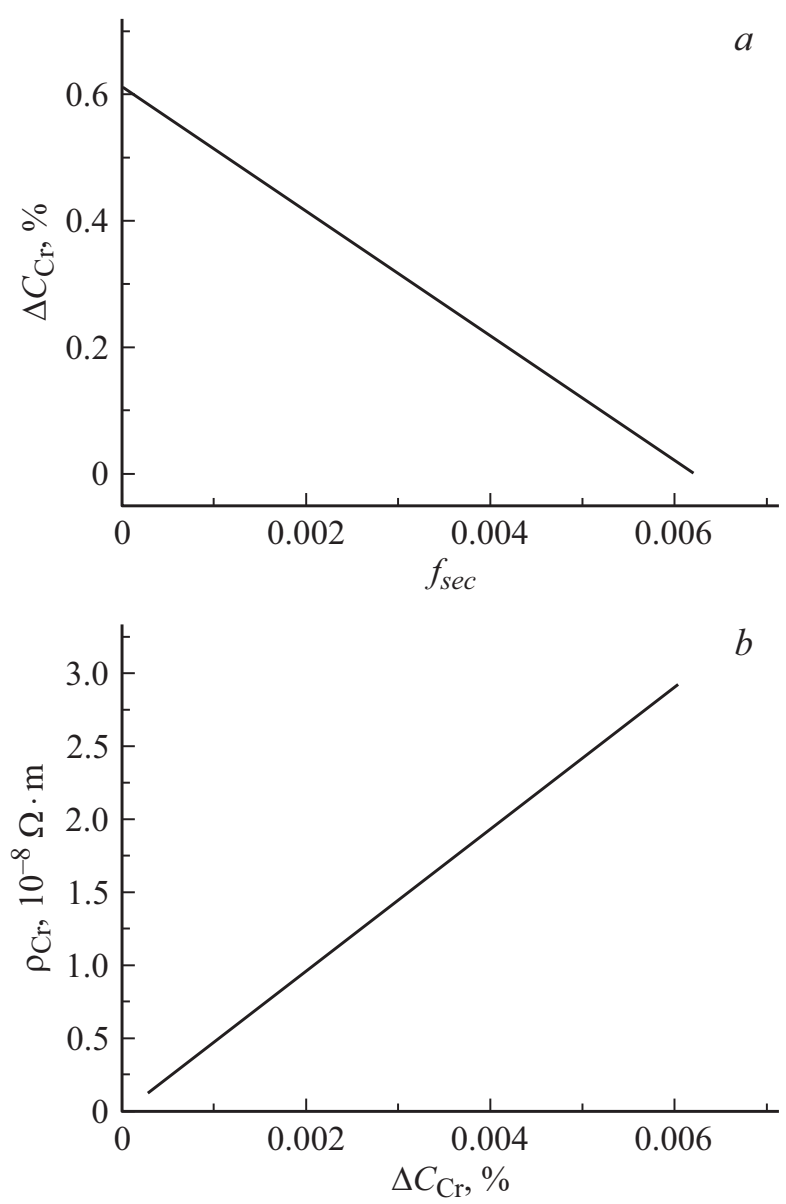

Pис. 3. Зависимость атомной концентрации $\Delta C_{\text {Cr }}$ растворенных в матрице атомов $\mathrm{Cr}$ от объемной доли $f_{\text {sec }}$ частиц вторичной фазы $(a)$, вклад растворенных в матрице атомов $\mathrm{Cr}$ $\rho_{\mathrm{Cr}}$ в удельное сопротивление сплава $\mathrm{Cu}-\mathrm{Cr}$ в зависимости от концентрации $\Delta C_{\mathrm{Cr}}$ атомов $\mathrm{Cr}$ в матрице $(b)$.

\subsubsection{2. Вклад частиц вторичной фазы}

Вклад частиц вторичной фазы в удельное сопротивление, согласно правилу (6), можно представить в виде

$$
\rho_{s e c}=\frac{m_{e} u_{F} f_{s e c}}{n_{a} e^{2}} \frac{3}{4 R^{3}}(R+r)^{2}
$$

так как, согласно формуле (10), объемная концентрация выделившихся частиц равна

$$
C_{V s e c}=\frac{N_{s e c}}{V_{s}}=\frac{3 f_{s e c}}{4 \pi R^{3}}
$$

При этом сечение рассеяния принято равным $S_{s e c} \approx \pi(R+r)^{2}$ поскольку с каждым атомом связана длина экранирования $r$. Увеличение объемной доли выделившихся частиц и уменьшение их размера приводит к увеличению удельного сопротивления (рис. 4).

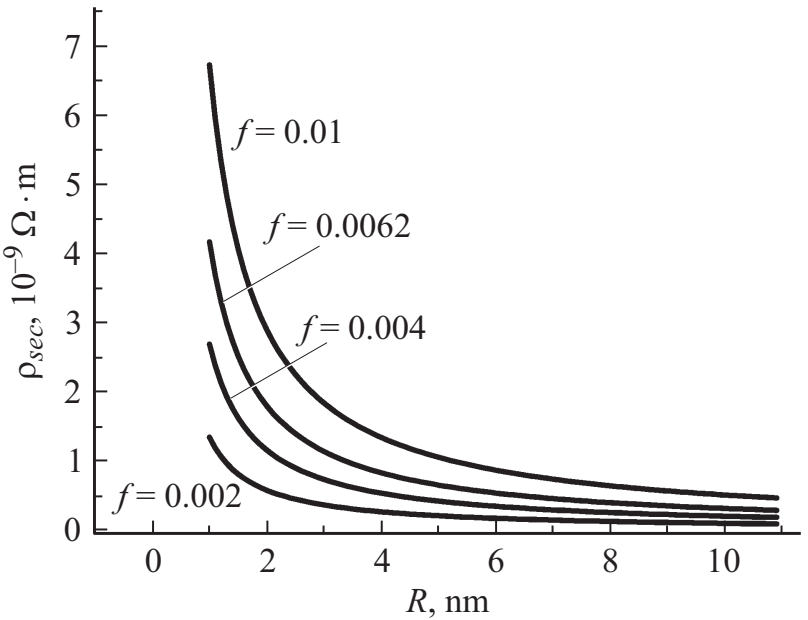

Рис. 4. Вклад в удельное сопротивление сплава $\mathrm{Cu}-\mathrm{Cr}$ выделившихся в результате термообработки частиц вторичной фазы в зависимости от их размера $R$ и объемной доли $f=f_{\mathrm{sec}}$.

\subsubsection{3. Вклад дислокаций}

Материалы, полученные методом ИПД, характеризуются большой плотностью дислокаций. Введение дислокаций в структуру материала способствует его упрочнению [14]. В то же время они повышают электрическое сопротивление образцов. Соответствующие оценки показывают, что вклад дислокаций в удельное сопротивление равен [35]

$$
\rho_{\text {disl }} \approx \frac{\hbar k^{2} l^{2} \rho_{t o t}}{9 n_{e} e^{2}}
$$

где $l^{2}$ - сечение ядра дислокации $(l=a / \sqrt[3]{4}, a-$ период кристаллической решетки $), k=2 \pi / \lambda-$ волновое число, $\lambda=h / m_{e} u_{F}$ - длина волны электрона, $\hbar-$ постоянная Планка. Зависимость приращения удельного сопротивления $\mathrm{Cu}$ от плотности дислокаций в нем представлена на рис. 5.

\section{2. Результаты анализа вклада структурных параметров в уровень прочностных характеристик и электропроводимость сплава Cu-0.5 wt.\% Cr, подвергнутого ИПД}

Представленные выше аналитические модели были применены для анализа прочности и электропроводности сплава $\mathrm{Cu}-0.5$ wt.\% Cr, подвергнутого ИПД, а также ИПД и последующей холодной прокатке и отжигу. В экспериментальной работе [5] показано, что число проходов РКУП не влияет на его электропроводность и составляет около 35\% IACS. При этом предел прочности после четырех проходов РКУП (состояние 4ЕСАР) был равен $\approx 460 \mathrm{MPa}$. Последующее применение холодной прокатки и отжига при $450^{\circ} \mathrm{C}$ в течение $1 \mathrm{~h}$ (состояние 
Таблица 1. Параметры микроструктурных состояний [37]

\begin{tabular}{c|c|c|c}
\hline Состояние & $D_{X R D}, \mathrm{~nm}$ & $\left\langle\varepsilon^{2}\right\rangle^{1 / 2}, \%$ & $\rho_{t o t}, 10^{14} \mathrm{~m}^{-2}$ \\
\hline 4ECAP & $39(3)$ & $0.240(5)$ & 8.33 \\
4ECAP+CR & $36(5)$ & $0.254(6)$ & 9.55
\end{tabular}

Таблица 2. Условные значения напряжений и деформаций, соответствующие пределам текучести $\left(\sigma_{Y}, \varepsilon_{Y}\right)$ и прочности $\left(\sigma_{u}, \varepsilon_{u}\right)[5]$

\begin{tabular}{c|c|c|c|c}
\hline Состояние & $\sigma_{Y}, \mathrm{MPa}$ & $\varepsilon_{Y}, \%$ & $\sigma_{u}, \mathrm{MPa}$ & $\varepsilon_{u}, \%$ \\
\hline 4ECAP & 450 & 3.8 & 460 & 4.3 \\
4ECAP+CR+A & 520 & 3.8 & 554 & 9.2
\end{tabular}

Таблица 3. Вклад различных механизмов упрочнения в напряжение течения и истинные значения напряжений, соответствующие пределам текучести и прочности сплава $\mathrm{Cu}$ $0.5 \mathrm{wt} . \% \mathrm{Cr}$, взятого в различных структурных состояниях

\begin{tabular}{c|c|c|c|c|c|c}
\hline Состояние & $\begin{array}{c}\sigma_{P}, \\
\mathrm{MPa}\end{array}$ & $\begin{array}{c}\sigma_{\text {sec }}, \\
\mathrm{MPa}\end{array}$ & $\begin{array}{c}\sigma_{\text {dislY }}, \\
\mathrm{MPa}\end{array}$ & $\begin{array}{c}\sigma_{\text {disl }}, \\
\mathrm{MPa}\end{array}$ & $\begin{array}{c}\sigma_{Y i}, \\
\mathrm{MPa}\end{array}$ & $\begin{array}{c}\sigma_{u i}, \\
\mathrm{MPa}\end{array}$ \\
\hline 4ECAP & 80.9 & - & 352 & 359 & 433 & 440 \\
4ECAP+CR+A & 80.9 & 270 & 149 & 152 & 500 & 503
\end{tabular}

4ЕСАР + CR +A) привело к увеличению предела прочности до $554 \mathrm{MPa}$, электропроводности - до 84\% IACS.

После РКУП в структуре сплава находились дислокации, плотность которых была вычислена по формуле [36]

$$
\rho_{0}=\frac{2 \sqrt{3}\left\langle\varepsilon^{2}\right\rangle^{1 / 2}}{D_{X R D} B},
$$

где $D_{X R D}$ - размер областей когерентного рассеяния, $\left\langle\varepsilon^{2}\right\rangle^{1 / 2}$ - величина среднеквадратичных упругих микроискажений кристаллической решетки, с учетом экспериментальных данных (табл. 1), приведенных в работе [37].

В напряжение течения $\sigma_{i}$ в процессе растяжения при комнатной температуре образцов сплава после 4 проходов РКУП вносят вклад напряжение Пайерлса $\sigma_{P}(1)$, дислокационное упрочнение $\sigma_{d i s l}(2)$ и твердорастворное упрочнение $\sigma_{c}(3)$ :

$$
\sigma_{i}=\sigma_{P}+\sigma_{c}+M \alpha G b \sqrt{\rho_{t o t}},
$$

где фактор Тейлора $M=3.06$. Исходя из инженерных кривых напряжение - деформация, полученных при растяжении ультрамелкозернистых образцов сплавов в различных состояниях, по формуле $\sigma_{i}=\sigma(1-\varepsilon)$, где $\sigma$ - условное напряжение, $\varepsilon-$ деформация (табл. 2), были рассчитаны истинные значения пределов текучести $\sigma_{Y i}$ и прочности $\sigma_{u i}$ образцов на растяжение (табл. 3 ).

Напряжение Пайерлса $\sigma_{P}(1)$ для $\mathrm{Cu}$, вычисленное при соответствующих значениях параметров $x=a / \sqrt{3}$,

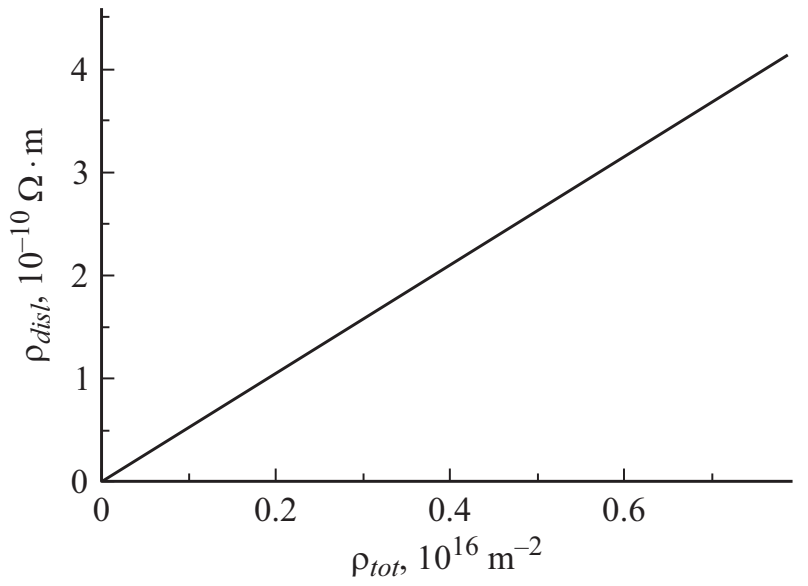

Рис. 5. Вклад в удельное сопротивление сплава $\mathrm{Cu}-\mathrm{Cr}$ дислокаций в зависимости от их плотности $\rho_{t o t}$.

$a=0.3615 \cdot 10^{-9} \mathrm{~m}, \quad d=b=0.256 \mathrm{~nm}, \quad v=0.343$, $G=42.1 \mathrm{GPa}$ оказалось равным $\sigma_{P}=80.9 \mathrm{MPa} \mathrm{в} \mathrm{случае}$ краевых дислокаций. В случае винтовых дислокаций $\sigma_{P}=770 \mathrm{MPa}$, что больше предела текучести и предела прочности. Поэтому было принято, что действие силы трения со стороны кристаллической решетки при движении испытывают краевые дислокации.

Атомы хрома были растворены в матрице. В соответствии с формулой (13) их концентрация составляла $\Delta C_{\mathrm{Cr}}=0.0061$. Оценка твердорастворного упрочнения (3) для медного сплава, содержащего $0.5 \mathrm{wt} . \% \mathrm{Cr}$, была проведена при значении параметра $\varepsilon_{s} \approx 0.6$, характерного для растворенных атомов никеля [17], в силу отсутствия данных для хрома, что привело к значению $\sigma_{c} \approx 0.48 \mathrm{MPa}$. Поскольку вклад твердорастворного упрочнения оказался мал, он в дальнейшем не был учтен. Таким образом, использование в расчетах значения, характерного для никеля вместо хрома, не является критичным.

Параметр $\alpha$ был вычислен, согласно формуле (17), с учетом известного из эксперимента истинного значения предела текучести $\sigma_{Y i}$ (табл. 3) и известного значения плотности дислокаций (табл. 1$), \alpha=0.37$. В момент достижения предела прочности при растяжении образцов сплава после 4 проходов РКУП плотность дислокаций достигла значения $8.66 \cdot 10^{14} \mathrm{~m}^{-2}$ как следует из той же формулы (17).

Согласно проведенным по формулам (6), (13), (14), (16) расчетам (рис. 3,5) с учетом модельного значения $\rho_{T \mathrm{Cu}}$ при комнатной температуре, вклад указанных дефектов в удельное сопротивление (табл. 4) привел к значению, равному $\rho=\rho_{\mathrm{Cr}}+\rho_{d i s l}+\rho_{T \mathrm{Cu}} \approx 4.66 \cdot 10^{-8} \Omega \cdot \mathrm{m}$, что соответствует 37\% IACS. Авторы работы [38] на основе измерения удельного сопротивления $\mathrm{Cu}$ до и после холодной обработки получили экспериментальную формулу, позволяющую оценить вклад дислокаций в удельное сопротивление: $\rho_{d i s l} \sim 2.3 \cdot 10^{-19} \rho_{t o t} \Omega \cdot \mathrm{cm}$. Учет указанной формулы приводит к значению 
$\rho=\rho_{\mathrm{Cr}}+\rho_{\text {disl }}+\rho_{T \mathrm{Cu}} \approx 4.68 \cdot 10^{-8} \Omega \cdot \mathrm{m}$ или $36.8 \% \mathrm{IACS}$, что согласуется с результатом, полученным с учетом формулы (16) и экспериментальным значением (небольшая разница объясняется погрешностью определения плотности дислокаций).

Микротвердость после четырех проходов РКУП и холодной прокатки с последующим отжигом при температуре $450^{\circ} \mathrm{C}$ в течение $1 \mathrm{~h}$ достигла максимального значения, что соответствует выделению частиц вторичной фазы [5]. Как увеличение температуры отжига, так и увеличение времени отжига при 450 ${ }^{\circ}$ приводят к уменьшению микротвердости [5], что соответствует увеличению размера частиц вторичной фазы. Максимальный радиус частиц, которые перерезаются дислокациями, был вычислен при значении параметра $\xi=0.015$ [15]. В рассматриваемом случае он оказался равным $r_{\text {max }} \approx 3.2 \mathrm{~nm}$.

После РКУП и последующей холодной прокатки плотность дислокаций возросла (табл. 1). Однако после отжига она обычно уменьшается. Как следует из проведенных расчетов (рис. 5), вклад дислокаций в удельное сопротивление пренебрежимо мал. Согласно вычислениям по формулам (6), (13)-(16) с учетом значений удельного сопротивления $\rho_{T \mathrm{Cu}} \approx 1.7 \cdot 10^{-8} \Omega \cdot \mathrm{m}$ и плотности дислокаций в состоянии 4ЕСАР $+\mathrm{CR}$ (табл. 1), зависимость удельного сопротивления $\rho=\rho_{\mathrm{Cr}}+\rho_{d i s l}+\rho_{T \mathrm{Cu}}+\rho_{\text {sec }}$ от объемной доли частиц вторичной фазы $f_{\text {sec }}$ имеет вид, представленный на рис. 6. Удельное сопротивление $84 \%$ IACS, которое соответствует экспериментальному значению, полученному авторами работы [5], достигается при значении радиуса выделений $R=r_{\max }$ и объемной доле частиц вторичной фазы, равной $f_{\text {sec }} \approx 0.0057$ (рис. 6). При этом в результате выделения частиц вторичной фазы концентрация атомов хрома в матрице уменьшилась. Объемной доле частиц вторичной фазы $f_{s e c} \approx 0.0057$, как следует из формулы (13) (рис. 3), соответствует концентрация атомов $\mathrm{Cr}$, равная $\Delta C_{\mathrm{Cr}}=5.1 \cdot 10^{-4}$. Тогда, согласно проведенным оценкам, вкладу каждого из дефектов кристаллической решетки в удельное сопротивление $\rho$ соответствуют значения, представленные в табл. 4. При этом $\rho_{d i s l}=5.01 \cdot 10^{-11} \Omega \cdot \mathrm{m}$. Учет даже максимального значения плотности дислокаций $\rho_{t o t}$, соответствующего состоянию 4ЕCAP $+\mathrm{CR}$, не оказал ощутимого влияния на величину удельного сопротивления. Значит, сделанный выбор объемной доли частиц вторичной фазы верен.

В данном случае напряжение течения включает вклад сформировавшихся при термообработке когерентных частиц вторичной фазы $\sigma_{s e c}$ :

$$
\sigma_{i}=\sigma_{P}+M \alpha G b \sqrt{\rho_{t o t}}+\sigma_{s e c}
$$

Оценка упрочнения когерентными частицами $\sigma_{s e c}$, проведенная согласно формуле (4) при значении радиуса частиц $R=r_{\max }$, привела к значению $\sigma_{s e c} \approx 270 \mathrm{MPa}$ (табл. 3). Из уравнения (18) с учетом значения напряжения $\sigma_{Y i}$ (табл. 3) следует, что начальная плот-

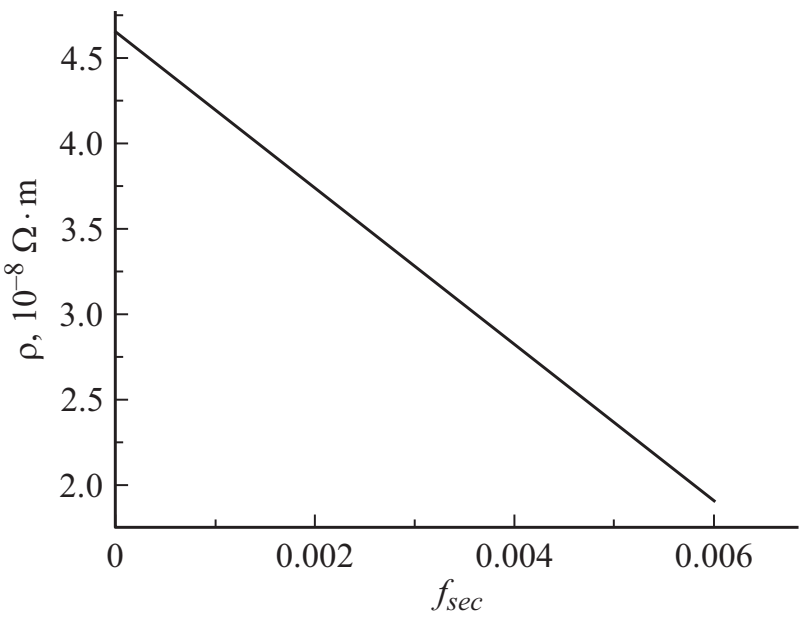

Рис. 6. Зависимость удельного сопротивления $\rho$ сплава $\mathrm{Cu}-$ $0.5 \mathrm{Cr}$, взятого в состоянии $4 \mathrm{ECAP}+\mathrm{CR}+\mathrm{A}$ от объемной доли частиц вторичной фазы $f_{\text {sec }}$.

ность дислокаций была равна $1.49 \cdot 10^{14} \mathrm{~m}^{-2}$. Соответственно вклад дислокаций в удельное сопротивление составил $\rho_{\text {disl }}=7.81 \cdot 10^{-12} \Omega \cdot \mathrm{m}$, который не повлиял на значение удельного сопротивления сплава (табл. 4). В момент достижения предела прочности $\sigma_{u i}$ (табл. 3) плотность дислокаций достигла значения $1.55 \cdot 10^{14} \mathrm{~m}^{-2}$. Плотности дислокаций в указанных состояниях по сравнению с таковыми в состояниях 4ЕCAР и 4ЕCAP+CR (табл. 1) уменьшились в результате отжига. Дислокационное упрочнение составило $\sigma_{d i s l Y}=149 \mathrm{MPa}$ и $\sigma_{\text {disl }}=152 \mathrm{MPa}$ соответственно. В состоянии после $4 \mathrm{ECAP}-\sigma_{d i s l Y}=352 \mathrm{MPa}$ и $\sigma_{d i s l u}=359 \mathrm{MPa}$. Однако напряжения, соответствующие пределу текучести $\sigma_{Y i}$ и прочности $\sigma_{u i}$ в состоянии 4ЕCAP $+\mathrm{CR}+\mathrm{A}$, выше (табл. 3) по сравнению с таковыми в состоянии 4ЕСАР благодаря упрочнению частицами вторичной фазы (табл. 3). Плотность дислокаций в момент достижения предела прочности увеличилась на $0.33 \cdot 10^{14} \mathrm{~m}^{-2}$ в состоянии 4ЕСАР, в состоянии 4ЕCAP $+\mathrm{CR}+\mathrm{A}-$ на $0.06 \cdot 10^{14} \mathrm{~m}^{-2}$. Частицы вторичной фазы препятствуют скольжению дислокаций, замедляя процесс их размножения и отдаляя момент достижения состояния насыщения, а значит, и предела прочности сплава.

Проведенный анализ электропроводности сплава $\mathrm{Cu}$ 0.5 wt.\% Cr, взятого в различных структурных состояниях, показал, что существенный вклад в его удельное сопротивление после четырех проходов РКУП, когда в структуре отсутствовали частицы вторичной фазы, вносят растворенные в матрице атомы $\mathrm{Cr}$, а также тепловые колебания атомов кристаллической решетки (табл. 4). В то же время, согласно проведенным по формуле (15) оценкам, вклад в удельное сопротивление сплава частиц вторичной фазы, сформировавшихся в результате холодной прокатки после четырех проходов РКУП и последующего отжига при температуре $450^{\circ} \mathrm{C}$ в течение $1 \mathrm{~h}$ оказался на два порядка меньше, а оставшихся 
Таблица 4. Расчетные значения удельных электросопротивлений, обусловленных дефектами кристаллической решетки, и удельного сопротивления $\rho$ сплава $\mathrm{Cu}-0.5 \mathrm{wt} . \% \mathrm{Cr}$ в различных структурных состояниях

\begin{tabular}{c|c|c|c|c|c}
\hline Состояние & $\rho_{\mathrm{Cr}}, \Omega \cdot \mathrm{m}$ & $\rho_{\text {disl }}, \Omega \cdot \mathrm{m}$ & $\rho_{\text {sec }}, \Omega \cdot \mathrm{m}$ & $\rho_{T \mathrm{Cu}}, \Omega \cdot \mathrm{m}$ & $\rho, \Omega \cdot \mathrm{m}$ \\
\hline 4ЕСАР & $2.96 \cdot 10^{-8}$ & $4.37 \cdot 10^{-11}$ & - & $1.70 \cdot 10^{-8}$ & $4.66 \cdot 10^{-8}$ \\
4ECAP+CR+A & $2.48 \cdot 10^{-9}$ & $7.81 \cdot 10^{-12}$ & $9.72 \cdot 10^{-10}$ & $1.70 \cdot 10^{-8}$ & $2.05 \cdot 10^{-8}$
\end{tabular}

в матрице растворенных атомов $\mathrm{Cr}$ на порядок меньше вклада тепловых колебаний кристаллической решетки (табл. 4). Выделение примесных атомов из раствора и формирование частиц вторичной фазы способствовало формированию наноструктурного материала, обладающего высокой электропроводностью и повышенными прочностными характеристиками.

\section{Заключение}

Прочность и электропроводность сплавов системы $\mathrm{Cu}-\mathrm{Cr}$ зависят от состояния их микроструктуры. В peзультате термообработки наноструктурных сплавов, полученных в результате ИПД, происходит выделение примесных атомов $\mathrm{Cr}$ из раствора с образованием частиц вторичной фазы, которые являются препятствиями на пути скользящих дислокаций. Такой процесс способствует формированию в таких материалах высокопрочных состояний, обладающих более высокой пластичностью. Дислокационное упрочнение в сплаве $\mathrm{Cu}-0.5 \mathrm{wt} . \% \mathrm{Cr}$ после четырех проходов РКУП и последующего отжига при температуре $450^{\circ} \mathrm{C}$ в течение $1 \mathrm{~h}$ оказалось меньше такового после четырех проходов РКУП на $203 \mathrm{MPa}$ В момент достижения предела прочности - на $207 \mathrm{MPa.}$ Упрочнение в результате выделения частиц вторичной фазы составило $270 \mathrm{MPa}$, что послужило причиной более высокой прочности в указанном состоянии, чем в состоянии после четырех проходов РКУП.

Увеличение размеров частиц вторичной фазы в результате выделения легирующих атомов привело к повышению электрической проводимости такого материала, так как вклад в удельное сопротивление растворенных атомов $\mathrm{Cr}$ уменьшился на порядок. Вклад частиц вторичной фазы оказался на порядок меньше вклада легирующих атомов. При этом вклад дислокаций в электропроводность был пренебрежимо мал.

Механические прочностные характеристики материала и его электропроводящие свойства оказываются взаимосвязанными. Анализ вклада различных структурных параметров объемных наноструктурных металлических материалов в их прочность и электропроводность выявил, что увеличение размера частиц вторичной фазы и их объемной доли приводит к повышению прочностных характеристик и в то же время способствует понижению удельного сопротивления таких материалов. Возрастание плотности дислокаций повышает прочность и практически не влияет на их электропроводимость.
Твердорастворное упрочнение пренебрежимо мало. В то же время легирующие атомы вносят значительный вклад в удельное сопротивление.

Представленные аналитические зависимости позволяют прогнозировать прочностные характеристики и электропроводность материала при известных значениях структурных параметров, или по известным значениям электропроводности и прочностных характеристик определять неизвестные значения структурных параметров.

Представленный метод исследования позволяет на основе предварительного прогнозирования планировать технологию обработки металлического материала для формирования требуемых прочностных и электропроводящих свойств, не прибегая к многочисленным трудоемким и дорогостоящим экспериментам.

Работа выполнена при финансовой поддержке Министерства образования и науки РФ в рамках реализации проекта № 16.1969.2017/4.6.

\section{Список литературы}

[1] Валиев Р.З., Александров И.В. Наноструктурные материалы, полученные интенсивной пластической деформацией. М.: Логос, 2000. $271 \mathrm{c}$.

[2] Мурашкин М.Ю., Кильмаметов А.Р., Валиев Р.З. // ФММ. 2008. Т. 106. № 1. C. 93-99. [Murashkin M.Yu., Kil'mametov A.R., Valiev R.Z. // Phys. Met. Metallogr. 2008. Vol. 106. N 1. P. 90-96.]

[3] An X.H., Lin Q.Y., Wu S.D., Zhang Z.F., Figueiredo R.B., Gao N., Langdon T.G. // Scripta Mater. 2011. Vol. 64. P. 954957.

[4] Zhao Y., Bingert J.F., Liao X., Cui B., Han K., Sergueeva A.V., Mukherjee A.K., Valiev R.Z., Langdon T.G., Zhu Y.T. // Adv. Mater. 2006. Vol. 18. P. 2949-2953.

[5] Wei K.X., Wei W., Wang F., Du Q.B., Alexandrov I.V., Hu J. // Mater. Sci. Engineer:: A. 2011. Vol. 528. N 3. P. 1478-1484.

[6] Islamgaliev R.K., Nesterov K.M., Bourgon J., Champion Y., Valiev R.Z. // J. Appl. Phys. 2014. Vol. 115. N 19. P. 194301. DOI: $10.1063 / 1.4874655$

[7] Howie A. // Philosophical Magazine. 1960. Vol. 5. N 51. P. 251-271. DOI: $10.1080 / 14786436008235840$

[8] Абрикосов А.А. Основы теории металлов. М.: Наука, 1987. $520 \mathrm{c}$.

[9] Кузнецов М.И. Основы электротехники. М.: Высшая школа, 1964. $560 \mathrm{c.}$

[10] Wei K.X., Wei W., Alexandrov I.V., Du Q.B., Hu J. // Advanc. Mater. Res. Vol. 391-392. 2012. P. 385-389.

[11] Lee S., Matsunaga H., Sauvage X., Horita Z. // Mater. Characterization. 2014. Vol. 90. P. 62-70. 
[12] Vinogradov A., Patlan V., Suzuki Y., Kitagawa K., Kopylov V.I. // Acta Mater. 2002. Vol. 50. N 7. P. 1639-1651.

[13] Peierls R. // Proc. Phys. Soc. 1940. Vol. 52. P. 34-37.

[14] Конрад X. В сб.: Сверхмелкое зерно в металлах / Под ред. Л.К. Гордиенко. М.: Металлургия, 1973. С. 206.

[15] Liu P., Kang B.X., Cao X.G., Huang J.L., Gu H.C. // J. Mater. Sci. 2000. Vol. 35. P. 1691-1694.

[16] Хаазен П. // Физическое металловедение. М.: Мир, 1968. C. 248-326.

[17] Флейшер Р., Хаббард У. В сб.: Структура и механические свойства металлов / Под ред. В.А. Алексеева. М.: Металлургия, 1967. С. 85.

[18] Штремель М.А. Прочность сплавов. Ч. II. Деформация. М.: МИСИС, 1997. 527 с.

[19] Гольдштейн М.И., Фарбер Б.М. Дисперсионное упрочнение стали. М.: Металлургия, 1979. 208 с.

[20] Эщби И.Ф. // Физика прочности и пластичности. М.: Металлургия, 1972. С. 88-107.

[21] Gerold V. In: Dislocations in Solids / Ed. by F.R.N. Nabarro, North Holland. 1979. Vol. 4. P. 220.

[22] Ardell A.J. // Metall. Trans. A. 1985. Vol. 16. P. 2131-2162.

[23] Хирм П.Б., Хэмпбри Ф.Джс. // Физика прочности и пластичности. М.: Металлургия, 1972. С. 158-186.

[24] Foreman A.J.E., Maki M.I. // Phil. Mag. 1966. Vol. 14. N 9. P. 911-924.

[25] Бардин Джсон // УФН. 1941. Т. ХХV. Вып. 1. С. 19-54.

[26] Сивухин Д.В. Общий курс физики. Термодинамика и молекулярная физика. Т. II. М.: Физматлит, 2005. 544 с.

[27] Займан Джс. Электроны и фононы. М.: ИЛ, 1962. 488 с.

[28] Блатт Ф. Физика электронной проводимости в твердых телах. М.: Мир, 1971. 470 с.

[29] Коллективный автор. Физика низких температур. СПб.: ИЛ, 1959. $452 \mathrm{c.}$

[30] Matthiessen A., Vogt C. // Philosophical Transactions - The Royal Society. 1864. Vol. 154. P. 167-200.

[31] Miyajima Y., Komatsu S.Y., Mitsuhara M., Hata S., Nakashima H., Tsuji N. // Philosophical Magazine. 2010. Vol. 90. N 34. P. 4475-4488.

[32] Botcharova E., Freudenberger J., Schultz L. // Acta Mater. 2006. Vol. 54. N 12. P. 3333-3341.

[33] Морозов А.И. Физика твердого тела. Электроны в кристалле. Металлы. Полупроводники. Диэлектрики. Магнетики. Сверхпроводники. М.: МИРЭА, 2008. 183 с.

[34] Марч Н., Кон В., Вашишта П., Лундквист С., Уильямс А., Барт У., Лэнг H. Теория неоднородного электронного газа. М.: Мир, 1987. 400 с.

[35] Harrison W.A. // J. Phys. Chem. Sol. 1958. Vol. 5. P. 44-46.

[36] Sanders P.G., Withey A.B., Weertman J.R. et al. // Mater. Sci. Eng. A. 1995. Vol. 204. P. 7-15.

[37] Alexandrov I.V., Sitdikov V.D., Abramova M.M., Sarkeeva E.A., Wei K.X., Wei W. // J. Mater. Engineer. Performance. 2012. Vol. 25. P. 4303-4309.

[38] Blevitt T.H., Coltman R.R., Redman J.K. // Phys. Rev. 1954. Vol. 93. N 4. P. 891.

DOI: https://doi.org/10.1103/PhysRev.93.891 